\title{
Effect of National Health Insurance Coverage on Adherence to Positive Airway Pressure Therapy in Obstructive Sleep Apnea Patients
}

\author{
Ji-Hwan Park, MD, Ji-Hun Kang, MD, Myeong-Gu Seo, MD, Ha-Nee Kwon, MD, \\ Sung-Dong Kim, MD, PhD, Kyu-Sup Cho, MD, PhD \\ Department of Otorhinolaryngology and Biomedical Research Institute, Pusan National University School of Medicine, Pusan National University Hospital, \\ Busan, Korea
}

Received: November 19, 2020

Revised: December 4, 2020

Accepted: December 21, 2020

Correspondence

Kyu-Sup Cho, MD, PhD

Department of Otorhinolaryngology and

Biomedical Research Institute,

Pusan National University School of Medicine,

Pusan National University Hospital,

179 Gudeok-ro, Seo-gu, Busan 49241, Korea

Tel +82-51-240-7824

Fax +82-51-246-8668

E-mail_choks@pusan.ac.kr

ORCID

Ji-Hwan Park

https://orcid.org/0000-0001-9301-6957

Ji-Hun Kang

https://orcid.org/0000-0002-8184-6512

Myeong-Gu Seo

https://orcid.org/0000-0003-2025-2769

Ha-Nee Kwon

https://orcid.org/0000-0002-6965-9589

Sung-Dong Kim

https://orcid.org/0000-0002-8436-5722

Kyu-Sup Cho

https://orcid.org/0000-0002-4381-6996

(c) This is an Open Access article distributed under the terms of the Creative Commons Attribution Non-Commercial License (https://creativecommons.org/licenses/by-nc/4.0) which permits unrestricted non-commercial use, distribution, and reproduction in any medium, provided the original work is properly cited.
Background and Objective Positive airway pressure (PAP) is considered a standard treatment for obstructive sleep apnea (OSA). Although PAP is effective in resolving upper airway collapse, a major challenge is maximizing adherence. Polysomnography and PAP have been included in Korean National Health Insurance (NHI) coverage since July 2018. This study was performed to investigate the adherence to PAP therapy according to the device manager after NHI coverage in OSA patients.

Methods Between July 2018 and May 2020, a total of 211 patients were prescribed PAP in a single tertiary hospital, and the PAP devices were managed by two companies. Among them, 187 patients constituted the final study population. Assessment of adherence was performed at 3 and 12 months after initiating PAP therapy. Good adherence was defined as using a PAP device for $\geq 4$ hours daily and on $\geq 70 \%$ of nights.

Results Ninety-three patients used the PAP machine obtained from device manager A and 94 patients used the PAP machine obtained from device manager B. During 90 days, the total good adherence rate was $66.8 \%(\mathrm{n}=125)$. The good adherence rate for device manager $\mathrm{A}$ was $66.7 \%$ $(62 / 93)$ and that for device manager B was $67.0 \%$ (63/94), which was not statistically different ( $\mathrm{p}=$ 0.959). However, the good adherence rate at 1 year after initiating PAP therapy was $38.7 \%(36 / 93)$ for device manager A and 58.5\% (55/94) for device manager B, which was statistically significant $(\mathrm{p}=0.007)$.

Conclusions The good adherence rate was decreased at 1 year after initiating PAP therapy despite NHI coverage. Furthermore, the device manager may play an important role in increasing the adherence rate in patients with OSA.

Sleep Med Res 2020;11(2):140-144

Key Words National health insurance; Continuous positive airway pressure; Sleep apnea, obstructive; Patient compliance; Health personnel.

\section{INTRODUCTION}

Obstructive sleep apnea (OSA) is a common disorder characterized by recurrent sleep-induced occlusion of the upper airway, leading to nocturnal hypoxemia, sleep fragmentation, and daytime hypersomnolence [1]. Furthermore, untreated OSA has been found to be an independent risk factor for cardiovascular morbidity, including hypertension, coronary heart disease, congestive heart failure, arrhythmias, pulmonary hypertension, stroke, and sudden death [2-4].

Positive airway pressure (PAP) has been regarded as the first-line therapy for patients with moderate and severe OSA after continuous PAP (CPAP) was first used by Sullivan et al. in the 
treatment of OSA in 1981 [5,6]. PAP therapy involves maintaining upper airway patency, improving alveolar ventilation, reducing the work of breathing, and eliminating hypoxemia [7]. As PAP is a self-administered treatment, its efficacy is critically dependent on the patient's willingness to use the device and apply the nasal mask during sleep. Therefore, adherence should be regarded as the main determinant for success of PAP therapy [8]. It has been reported that the adherence rate of PAP varies between 29-83\% [9].

Polysomnography (PSG) and PAP therapy have been included in Korean National Health Insurance (NHI) coverage since July 2018. The revised NHI coverage is expected to make PAP treatment more accessible to patients by reducing the cost of PAP devices and masks to $20 \%$ of the original levels for insured patients, thereby potentially improving the adherence to PAP therapy. Many companies rent out the PAP machine for a period of time and have their own differentiated strategy or service for OSA patients. Device managers of each company can help the patients to improve PAP adherence by close monitoring of physiological variables, such as air leak, and rapid troubleshooting of potential problems.

The purpose of this study was to investigate the adherence to PAP therapy at the adaptation period of 90 days and 1 year after treatment initiation according to the device manager after $\mathrm{NHI}$ coverage in patients with OSA.

\section{METHODS}

A total of 211 patients, who were prescribed PAP between July 2018 and May 2020 in a single tertiary hospital, were recruited and reviewed retrospectively. The patients should have experienced significant clinical symptoms, such as snoring, sleep apnea, morning headaches, tiredness, and daytime sleepiness and should have been diagnosed with OSA, with an apnea-hypopnea index $(\mathrm{AHI}) \geq 5$ events/h. The patients who had factors that might have an influence on the adherence to PAP therapy, such as severe deviated nasal septum, chronic rhinosinusitis, and previous history of surgery or severe cardiopulmonary condition, were excluded. Patient demographics, medical history, body mass index (BMI) $\left(\mathrm{kg} / \mathrm{m}^{2}\right)$, and pretreatment PSG results were reviewed. Disease severity according to the $\mathrm{AHI}$ was noted as mild $(5 \leq \mathrm{AHI}<15)$, moderate $(15 \leq \mathrm{AHI}<30)$, and severe $(\mathrm{AHI} \geq$ 30) OSA.

All patients were oriented to PAP during the adaptation period of 90 days in Korean NHI coverage. After this period, patients who showed good adherence could be included under insurance coverage. The criteria for good adherence were identified as using a PAP device regularly for more than 4 hours/night over $>70 \%$ of the recorded period. Adherence to PAP therapy was documented via downloading the "mask-on time tracking" data from PAP devices.
PAP devices were managed by two different companies that were allocated randomly. Objective assessment of adherence was performed at the adaptation period of 90 days and 1 year after treatment initiation according to the device manager. PAP managers of each company assisted the patients to improve PAP adherence by close monitoring and rapid troubleshooting of potential problems, such as mask discomfort, air leak, and skin irritation.

The data are presented as means \pm standard deviation or as numbers of patients and percentages. Statistical significance was assessed using the chi-square test or Student's t-test in IBM SPSS (version 19, SPSS Statistics/IBM Corp., Armonk, NY, USA). A $\mathrm{p}$-value $<0.05$ was considered to indicate statistical significance.

This study protocol was approved by the Institutional Review Board of Pusan National University Hospital (H-2012-005-097). However, as it was a retrospective chart review study, written informed consent was not obtained from each patient prior to the patient's participation in the study.

\section{RESULTS}

During the study period, 211 patients who had OSA detected by PSG were treated in this institution. Twenty-four patients (11.4\%) were excluded from this analysis because of PAP use less than 1 year, use of a purchased PAP device without NHI coverage, or loss to follow-up. One hundred and eighty-seven patients constituted the final study population, in which PAP adherence was analyzed (Fig. 1).

Ninety-three patients used the PAP machine obtained from device manager A and 94 patients used the PAP machine obtained from device manager B. There were 75 (80.7\%) male patients among the patients who used the PAP machine obtained from device manager A, and 77 male patients (81.9\%) among those who used the PAP machine obtained from device manager B. Mean age and BMI of patients who used the PAP machine obtained from device managers A and B were $50.1 \pm 12.5$ years and $26.9 \pm 4.1$, and $52.9 \pm 11.7$ years and $28.0 \pm 6.9$, respectively. Twelve (6.4\%) patients had mild, 52 (27.8\%) had moderate, and 123 (65.8\%) had severe OSA based on the severity of sleep respiratory disturbance. There were no significant differences in sex, age, BMI, and AHI between patients who used the PAP machine obtained from device managers A and B (Table 1).

During the adaptation period for 90 days, the total good adherence rate of PAP therapy was $66.8 \%(n=125)$. The total good adherence rate for device manager $\mathrm{A}$ was $66.7 \%$ (62/93) and that for device manager B was $67.0 \%$ (63/94), which was not statistically different $(\mathrm{p}=0.959)$. Although the good adherence rate was higher in severe $\mathrm{AHI}$ patients than in mild to moderate $\mathrm{AHI}$ patients, there was no significant difference between device managers A and B according to the AHI severity (Table 2).

The reasons for discontinuing the use of a PAP machine were 
determined by a telephone survey. The nasal mask discomfort was the most common reason, followed by personal issue and medical problem. There was no significant difference in reasons for non-adherence between device managers A and B (Table 3).

After the adaptation period, the total good adherence rate at 1 year after initiating PAP therapy was $48.6 \%$ (91/187). Although the good adherence rate of PAP therapy was correlated with higher AHI, PAP adherence was not significantly different in patients with mild and moderate OSA between device managers $A$ and B. However, good adherence rates for device manager B were $38.3 \%$ (36/94) and 58.5\% (55/94) and those for device manager A were $22.6 \%(21 / 93)$ and $38.7 \%$ (36/93) in patients with severe and total AHI, respectively. Good adherence rates for device man-

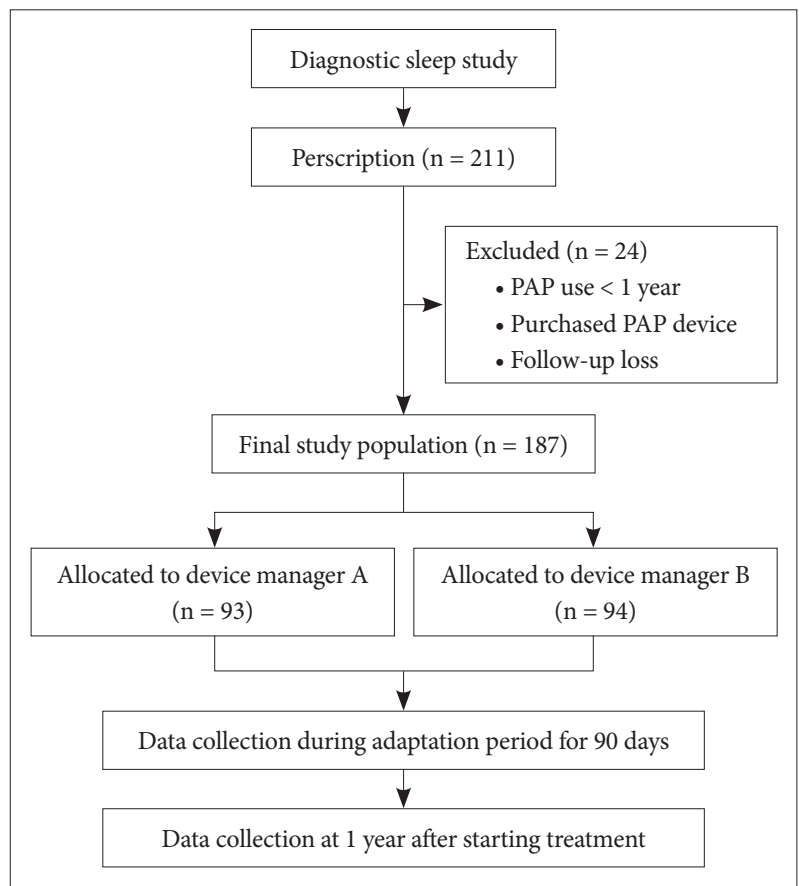

Fig. 1. Flow diagram of the study protocol. Of the 211 patients who underwent polysomnography for obstructive sleep apnea, 187 patients constituted the final study population, in which PAP adherence was analyzed. PAP: positive airway pressure.

Table 1. Demographic characteristics

\begin{tabular}{lccc}
\hline & $\begin{array}{c}\text { Manager A } \\
(\mathrm{n}=93)\end{array}$ & $\begin{array}{c}\text { Manager B } \\
(\mathrm{n}=94)\end{array}$ & p-value \\
\hline Male & $75(80.7)$ & $77(81.9)$ & 0.820 \\
Age (years) & $50.1 \pm 12.5$ & $52.9 \pm 11.7$ & 0.120 \\
BMI (kg/m²) & $26.9 \pm 4.1$ & $28.0 \pm 6.9$ & 0.170 \\
AHI (events/h) & $42.5 \pm 24.0$ & $44.4 \pm 21.6$ & 0.584 \\
$\quad$ Mild & $6(6.5)$ & $6(6.4)$ & 1.000 \\
Moderate & $30(32.3)$ & $22(23.4)$ & 0.194 \\
Severe & $57(61.3)$ & $66(70.2)$ & 0.220 \\
\hline
\end{tabular}

Data are expressed as the number (percentage) except age, BMI, and AHI (means \pm standard deviation).

AHI: apnea-hypopnea index, BMI: body mass index. ager B were significantly higher than those for device manager A in patients with severe and total AHI ( $p=0.026$ and $p=0.007$, respectively) (Table 4). Furthermore, common barriers to longterm adherence to PAP therapy assessed by phone calls were inconvenience of daily PAP use, removing the PAP early in the night, and dry mouth.

\section{DISCUSSION}

OSA has been managed with medication, positional therapy, oral appliances, or upper airway surgery, but PAP is the most effective treatment that involves maintenance of a positive pharyngeal transmural pressure in the upper airway during sleep [10]. Although PAP is highly effective in preventing upper airway collapse, major clinical challenge is maximizing PAP adherence because the effectiveness of the treatment is closely related to the duration and frequency of PAP device usage. However, a previous study showed that only $46 \%$ of all OSA patients treated with CPAP used PAP therapy for at least 4 hours on at least $70 \%$ of all nights [11]. Although extensive education, consis-

Table 2. Adherence rate during adaptation period for 90 days

\begin{tabular}{lccc}
\hline \multicolumn{1}{c}{ AHI } & $\begin{array}{c}\text { Manager A } \\
(\mathrm{n}=93)\end{array}$ & $\begin{array}{c}\text { Manager B } \\
(\mathrm{n}=94)\end{array}$ & p-value \\
\hline Mild & $5(5.4)$ & $4(4.3)$ & 0.747 \\
Moderate & $18(19.4)$ & $17(18.1)$ & 0.824 \\
Severe & $39(41.9)$ & $42(44.7)$ & 0.705 \\
Total & $62(66.7)$ & $63(67.0)$ & 0.959 \\
\hline
\end{tabular}

Data are expressed as the patients' number (percentage).

AHI: apnea-hypopnea index.

Table 3. Non-adherent reasons during adaptation period for 90 days

\begin{tabular}{lccc}
\hline \multicolumn{1}{c}{ Variables } & $\begin{array}{c}\text { Manager A } \\
(\mathrm{n}=31)\end{array}$ & $\begin{array}{c}\text { Manager B } \\
(\mathrm{n}=31)\end{array}$ & p-value \\
\hline No effect & 3 & 2 & 1.000 \\
Nasal mask discomfort & 16 & 18 & 0.799 \\
Change of treatment & 1 & 2 & 1.000 \\
Medical problem & 3 & 4 & 1.000 \\
Personal schedule & 8 & 5 & 0.534 \\
\hline \multicolumn{5}{c}{ AHI } & Manager A & Manager B \\
Table 4. Adherence rate at 1 year after PAP therapy & \\
\hline \multicolumn{5}{c}{ (n=93) } & (n = 94) & -value \\
\hline Mild & $3(3.2)$ & $4(4.3)$ & 1.000 \\
Moderate & $12(12.9)$ & $15(16.0)$ & 0.678 \\
Severe & $21(22.6)$ & $36(38.3)$ & 0.026 \\
Total & $36(38.7)$ & $55(58.5)$ & 0.007 \\
\hline
\end{tabular}

Data are expressed as the patients' number (percentage).

AHI: apnea-hypopnea index, PAP: positive airway pressure. 
tent follow-up, and regular feedback can achieve CPAP compliance rates of $>85 \%$ over 6 months [12], over $50 \%$ of patients commencing CPAP in the general population may not be using CPAP 1 year later [13].

Adherence to PAP therapy is influenced by many factors, including severity of OSA, side effects, therapeutic response, claustrophobia, patient's perception of disease seriousness, family support, and cost [14]. Increased air leak with auto-PAP therapy is associated with reduced adherence [15], and general management with interventions, such as heated humidification, mask optimization, and topical nasal therapy, improves the adherence [16]. Adherence can also be significantly affected by timely interventions by health professionals, such as physicians and device managers. Our previous study showed that there were statistically significant differences in the adherence to PAP therapy according to the doctor and device manager [17].

The cost of PAP therapy has recently been covered by the Korean NHI in patients with moderate-to-severe OSA or in patients with mild OSA associated with either symptoms (daytime sleepiness, insomnia, impaired cognition, and mood disorders) or comorbidities (hypertension, ischemic heart disease, and history of stroke). Coverage of PAP is initially limited to 90 days during the adaptation period. After that period, only patients showing good adherence ( $>4 \mathrm{~h} /$ night on $70 \%$ of nights) can use the PAP machine under NHI coverage. Economic factors could be important factors for good adherence to PAP therapy. Therefore, this study was designed to investigate the adherence to PAP therapy after Korean NHI coverage. Furthermore, we investigated whether the device manager has an effect on the adherence to PAP therapy in patients with OSA.

In this study, the total good adherence rate irrespective of the AHI severity was $66.8 \%$ at 90 days and $48.6 \%$ at 1 year after initiating PAP therapy. Furthermore, we found that OSA severity based on the AHI was associated with PAP compliance, and that as the disease worsened, the adherence increased. The adherence rate in our study was higher than that in previous studies in South Korea, which showed an adherence rate ranging from 30\% to $50 \%$ [18-21]. Therefore, NHI coverage had a positive effect on the adherence to PAP therapy in OSA patients. Although various factors are associated with adherence to PAP therapy, mask discomfort was the most common cause of discontinuing PAP therapy during the adaptation period. A PAP mask should fit the face snugly to avoid air leaks, but it should not be so tight that it feels uncomfortable or causes pain or irritation [22]. Although there were no differences between managers A and B in terms of their career, age, and gender, they dealt different PAP devices provided by different companies. However, there was no significant difference in the adherence rate between device managers $\mathrm{A}$ and $\mathrm{B}$ according to the AHI severity at 90 days, suggesting that the type of PAP device and company are not correlated with the adherence rate.

After the adaptation period, the good adherence rate was de- creased to $48.6 \%$ at 1 year after initiating PAP therapy despite NHI coverage. Furthermore, there was a statistically significant difference in the good adherence rate for PAP therapy between the two device managers, which was influenced by OSA severity. Patients with severe OSA tended to be more affected by the device manager than patients with mild-moderate OSA. These findings highlight the need for a good device manager to detect early and solve the problems related to PAP use by close monitoring because severe OSA patients have more potential problems [22,23]. Physicians should monitor for compliance and should follow-up their patients closely especially during the adaptation period of PAP therapy to ensure long-term success [24]. A device manager provides a patient with close monitoring of objective efficacy and usage data, early identification of difficulties with PAP use, and rapid troubleshooting of potential problems. Therefore, systematic education and management of PAP users, immediate problem solving, and high quality service after the adaptation period may underlie the higher adherence rates for device manager $\mathrm{B}$.

A limitation of the current study is that the findings were obtained from a single tertiary center. Therefore, the results cannot be extrapolated to other sleep disorders centers in South Korea. Moreover, data about the outcomes of physiological parameters were not collected. The longer term adherence rate can be expected as this result is followed closely over time.

In conclusion, NHI coverage had a significant impact on the adherence rate for PAP therapy in OSA patients. However, the good adherence rate was decreased at 1 year after initiating PAP therapy despite NHI coverage. Furthermore, the device manger may be an important factor for improving PAP adherence in patients with OSA.

\section{Acknowledgments}

This work was supported by the year 2020 clinical research grant from Pusan National University Hospital.

\section{Conflicts of Interest}

The authors have no financial conflicts of interest.

\section{Authors' Contribution}

Conceptualization: Cho KS. Data curation: Kang JH. Formal analysis: Kang JH. Funding acquisition: Cho KS. Investigation: Park JH. Methodology: Kim SD. Project administration: Kim SD. Resources: Kim SD. Software: Seo MG. Supervision: Cho KS. Validation: Cho KS. Visualization: Kwon HN. Writing_original draft: Park JH. Writing_review \& editing: Park JH, Cho KS.

\section{REFERENCES}

1. Seo MY, Lee SH. Compliance with continuous positive airway pressure in patients with obstructive sleep apnea. Sleep Med Res 2020;11:7-14.

2. Nieto FJ, Young TB, Lind BK, Shahar E, Samet JM, Redline S, et al. Association of sleep-disordered breathing, sleep apnea, and hypertension in a large community-based study. Sleep Heart Health Study. JAMA 2000;283:1829-36.

3. Gottlieb DJ, Yenokyan G, Newman AB, O'Connor GT, Punjabi NM, Quan SF, et al. Prospective study of obstructive sleep apnea and inci- 
dent coronary heart disease and heart failure: the sleep heart health study. Circulation 2010;122:352-60.

4. Redline S, Yenokyan G, Gottlieb DJ, Shahar E, O’Connor GT, Resnick HE, et al. Obstructive sleep apnea-hypopnea and incident stroke: the sleep heart health study. Am J Respir Crit Care Med 2010;182:269-77.

5. Rotenberg BW, Murariu D, Pang KP. Trends in CPAP adherence over twenty years of data collection: a flattened curve. J Otolaryngol Head Neck Surg 2016;45:43.

6. Sullivan CE, Issa FG, Berthon-Jones M, Eves L. Reversal of obstructive sleep apnoea by continuous positive airway pressure applied through the nares. Lancet 1981;1:862-5.

7. Donovan LM, Boeder S, Malhotra A, Patel SR. New developments in the use of positive airway pressure for obstructive sleep apnea. J Thorac Dis 2015;7:1323-42.

8. Collard P, Pieters T, Aubert G, Delguste P, Rodenstein DO. Compliance with nasal CPAP in obstructive sleep apnea patients. Sleep Med Rev 1997;1:33-44.

9. Weaver TE, Sawyer AM. Adherence to continuous positive airway pressure treatment for obstructive sleep apnoea: implications for future interventions. Indian J Med Res 2010;131:245-58.

10. Weaver TE, Mancini C, Maislin G, Cater J, Staley B, Richard JR, et al. Continuous positive airway pressure treatment of sleepy patients with milder obstructive sleep apnea: results of the CPAP Apnea Trial North American Program (CATNAP) randomized clinical trial. Am J Respir Crit Care Med 2012;186:677-83.

11. Kribbs NB, Pack AI, Kline LR, Smith PL, Schwartz AR, Schubert NM, et al. Objective measurement of patterns of nasal CPAP use by patients with obstructive sleep apnea. Am Rev Respir Dis 1993;147:887-95.

12. Sin DD, Mayers I, Man GC, Pawluk L. Long-term compliance rates to continuous positive airway pressure in obstructive sleep apnea: a population-based study. Chest 2002;121:430-5.

13. Stepnowsky CJ Jr, Moore PJ. Nasal CPAP treatment for obstructive sleep apnea: developing a new perspective on dosing strategies and compliance. J Psychosom Res 2003;54:599-605.

14. Fox N, Hirsch-Allen AJ, Goodfellow E, Wenner J, Fleetham J, Ryan CF, et al. The impact of a telemedicine monitoring system on positive air- way pressure adherence in patients with obstructive sleep apnea: a randomized controlled trial. Sleep 2012;35:477-81.

15. Valentin A, Subramanian S, Quan SF, Berry RB, Parthasarathy S. Air leak is associated with poor adherence to autoPAP therapy. Sleep 2011; 34:801-6.

16. Ballard RD, Gay PC, Strollo PJ. Interventions to improve compliance in sleep apnea patients previously non-compliant with continuous positive airway pressure. J Clin Sleep Med 2007;3:706-12.

17. Kim SL, Kim JA, Kim DS, Jo MG, Kim SD, Cho KS. Effect of doctor and device manager on adherence with positive airway pressure therapy in obstructive sleep apnea patients. J Rhinol 2018;25:26-31.

18. Kim JH, Kwon MS, Song HM, Lee BJ, Jang YJ, Chung YS. Compliance with positive airway pressure treatment for obstructive sleep apnea. Clin Exp Otorhinolaryngol 2009;2:90-6.

19. Choi JA, Yoon IY, Han EG, Lee S. Subjective and objective CPAP compliance in patients with obstructive sleep apnea. Sleep Med Res 2011;2: 63-8.

20. Han EK, Yoon IY, Chung SH. The compliance and effect of CPAP in obstructive sleep apnea syndrome. Sleep Med Psychophysiol 2006;13: $52-8$.

21. Choi JB, Lee SH, Jeong DU. Compliance of nasal continuous positive airway pressure in patients with obstructive sleep apnea syndrome. Sleep Med Psychophysiol 2006;13:27-32.

22. Genta PR, Kaminska M, Edwards BA, Ebben MR, Krieger AC, Tamisier $\mathrm{R}$, et al. The importance of mask selection on continuous positive airway pressure outcomes for obstructive sleep apnea. An Official American Thoracic Society Workshop Report. Ann Am Thorac Soc 2020;17:1177-85.

23. Jacobsen AR, Eriksen F, Hansen RW, Erlandsen M, Thorup L, Damgård $\mathrm{MB}$, et al. Determinants for adherence to continuous positive airway pressure therapy in obstructive sleep apnea. PLoS One 2017;12:e0189614.

24. Schwab RJ, Badr SM, Epstein LJ, Gay PC, Gozal D, Kohler M, et al. An official American Thoracic Society statement: continuous positive airway pressure adherence tracking systems. The optimal monitoring strategies and outcome measures in adults. Am J Respir Crit Care Med 2013;188:613-20. 